\title{
Incidental Kidney Stone Diagnosed During Laparoscopy-Assisted Vaginal Hysterectomy
}

\author{
Jennifer Schmitt, DO, Bradford Fenton, MD, PhD \\ Department of Obstetrics and Gynecology, Mayo Clinic, Rochester, Minnesota (Dr. Schmitt). \\ Department of Obstetrics and Gynecology, SUMMA Health Systems, Akron, Ohio (both authors).
}

\begin{abstract}
Introduction: Hysterectomies are the second most common major surgical procedure among women in their reproductive years. Ureteral injury is a known complication of this surgery. Intraoperative cystoscopy is not yet used routinely to evaluate the ureteral function before concluding a benign hysterectomy.

Case Description: A 40-year-old woman had a laparoscopy-assisted vaginal hysterectomy with intraoperative cystoscopy that noted unilateral absence of ureteral dye spill. Urology was consulted and diagnosed an incidental, complete, obstructing kidney stone in the ureter, which was removed and stented during an exploratory laparotomy.
\end{abstract}

Conclusion: Although the chance of ureteral obstruction secondary to a kidney stone is small, either during or after gynecologic surgery, this case suggests that it should remain on the list of differential diagnoses.

Key Words: Cystoscopy, Hysterectomy, Kidney stone, Laparoscopy, Ureteral obstruction.

\footnotetext{
Citation Schmitt J, Fenton B. Incidental kidney stone diagnosed during laparoscopy-assisted vaginal hysterectomy. CRSLS e2014.00248. DOI: 10.4293/JSLS.2014.00248.

Copyright (c) 2015 by SLS, Society of Laparoendoscopic Surgeons. This is an open-access article distributed under the terms of the Creative Commons Attribution-Noncommercial-ShareAlike 3.0 Unported license, which permits unrestricted noncommercial use, distribution, and reproduction in any medium, provided the original author and source are credited.

Financial disclosures: None reported.

Presented at the American Urogynecologic Society's 3rd Annual Scientific Meeting, October 3-6, 2012, Chicago, Illinois. Poster presentation.

Address correspondence to: Jennifer Schmitt, DO, Department of Obstetrics and Gynecology, Mayo Clinic, 200 First Street SW, Rochester, MN 55905, USA. Telephone:

612-703-5264. E-mail: schmitt.jennifer@mayo.edu
}

\section{INTRODUCTION}

The Centers for Disease Control estimates that approximately 600,000 hysterectomies are performed annually in the United States, and it is the second most common major surgical procedure among women in their reproductive years. ${ }^{1}$

The potential risks of morbidity associated with major pelvic surgery are numerous and include infection, hemorrhage, thromboembolism, and damage to surrounding structures, most notably the bowel, bladder, and ureters. Mortality is a rare outcome., ${ }^{2,3}$

The short- and-long term morbidities associated with ureteral injury or obstruction are significant and include pain, loss of kidney function, anxiety, depression, adverse effects on quality of life, additional surgeries, and either temporary or permanent loss of employment. ${ }^{3}$ The use of intraoperative cystoscopy to detect ureteral injury during major gynecologic surgeries has remained controversial, and despite multiple studies showing its benefit, the procedure is not yet routinely performed during all hysterectomies. In 1999, Ribeiro et $\mathrm{al}^{4}$ first reported their use of cystoscopy after laparoscopic hysterectomy, which enabled intraoperative detection and immediate repair of ureteral obstruction caused by sutures in 4 of 118 patients. Furthermore, Jelovsek et al ${ }^{5}$ reported a 5 -fold increased detection rate of ureteral injury with routine intraoperative use of cystoscopy, suggesting that it can detect injuries that otherwise may be overlooked and allow surgical repair with minimal inconvenience to the patient.

Intraoperative cystoscopy performed after a hysterectomy can reveal a ureteral injury or obstruction, with an absence of urine spilling from the ureteral orifice after an intravenous infusion. This finding can be associated with a suture or another type of iatrogenic blockage of a ureter. However, an additional etiology of ureteral obstruction, albeit rare, is an incidental intraoperative 
finding of an obstructing kidney stone. We present such a case after receiving a waiver from our Institutional Review Board.

\section{CASE DESCRIPTION}

A 40-year-old woman gravida 4, para 4 presented with 8 months of menometrorrhagia, with either spotting or heavy bleeding nearly every day. The patient was otherwise healthy, with a medical history significant only for asthma. Her surgical history included 2 cesarean deliveries and a bilateral tubal ligation.

Pelvic examination revealed a uterine size equivalent to 10 weeks' gestational age with a normal-appearing cervix. A transvaginal ultrasound confirmed a fibroid uterus measuring $10 \times 7 \times 6 \mathrm{~cm}$. An endometrial biopsy and Papanicolaou test were both negative for malignant disease.

Conservative treatment of the vaginal bleeding with nonsteroidal anti-inflammatory medications and progesterone therapy had been unsuccessful. The patient desired definitive surgical management, and the decision was made to proceed with a laparoscopy-assisted vaginal hysterectomy (LAVH) with an intraoperative cystoscopy to follow. The LAVH portion of the procedure was successfully completed without complication.

The cystoscopy was then performed after indigo carmine was intravenously injected. The bladder was intact and appeared grossly normal. The right ureteral orifice showed a steady flow of dye. However, the left ureter did not spill. An attempt was made to introduce a whistle-tip catheter in the left ureter, but it failed to pass beyond $3 \mathrm{~cm}$.

Subsequent evaluation was conducted in an intraoperative consultation with urology. The urologist attempted to pass a Glidewire (Terumo Medical Corporation, Somerset, New Jersey) and a 5-French Pollack catheter separately. The attempt with each was unsuccessful, with an obvious obstruction of unknown etiology noted in the left ureter at an approximate level just interior to the pelvic brim. When the catheter and guidewire were used in combination, however, the urologist was able to pass the catheter to a depth of $20 \mathrm{~cm}$. A pyelogram was then performed that revealed a $1.5-$ to 2 -cm defect in the proximal ureter, and a stent was successfully placed with a great deal of difficulty.

The procedure was converted to a laparotomy because of the very unusual clinical picture and the unknown etiology of the ureteral obstruction. The left ureter was pal- pated to the pelvic brim and noted to be normal. Attention was then turned to the renal pelvis, where, at the junction of the renal pelvis and ureter, the stent was palpated and a firm mass was noted. The ureter was incised, and an obstructing almond-shaped stone was visualized and removed. The stent was left in place. The ureter was repaired and a closed drain was placed before surgical closure. The patient had an uneventful postoperative course and was discharged home on postoperative day 3 with the stent and drain in place.

\section{DISCUSSION}

Renal stones are a relatively common condition, and if they are large enough, they can cause obstruction of the urinary tract during migration. The prevalence of renal stones was estimated to be $3.8 \%$ in 1980 and rose to $5.2 \%$ by 1994 . This increase was independent of sex and race. 6,7

This patient's intraoperative course was perplexing, as initially both the gynecologist and the urologist were unable to advance the stent. Only after using the guidewire and Pollack catheter together was the urologist able to place the stent at $20 \mathrm{~cm}$. It is our opinion that use of the guidewire and catheter in combination pushed the stone proximally from where the initial attempt at stenting halted to the final $20-\mathrm{cm}$ mark. Even though the stent was passed to $20 \mathrm{~cm}$, a 1.5 - to 2 -cm defect was still noted on a pyelogram, suggesting that the stent had not passed the obstruction or reached the renal pelvis, necessitating further investigation by laparotomy.

When we discussed this case at its conclusion, our urology colleagues suggested that a preoperative computed tomographic (CT) scan would have shown the renal stone and may have decreased the diagnostic uncertainly during surgery, which unfortunately led to a laparotomy. Although this opinion is certainly valid in this particular case, it is a rare finding. CT scans are not indicated as a routine preoperative screening modality for gynecologic patients undergoing benign hysterectomy, as they increase both radiation exposure and cost.

We found no published reports of another case of a kidney stone that completely obstructed a ureter during a routine intraoperative cystoscopy after a laparoscopic hysterectomy. Certainly, the most common reason for perioperative ureteral obstruction is iatrogenic injury. Although the incidence of complete ureteral obstruction secondary to a kidney stone is rare, whether during or after gynecologic surgery, this case suggests that the 
possibility should remain on the list of differential diagnoses.

\section{References:}

1. Clarke-Pearson C, Geller E. Complications of hysterectomy. Obstet Gynecol. 2013;121:3:654-673.

2. Makinen J, Johansson J, Tomas C, et al. Morbidity of 10110 hysterectomies by type of approach. Hum Reprod. 2001;16: $1473-1478$.

3. Gilmour DT, Das S, Flowerdew G. Rates of urinary tract injury from gynecologic surgery and the role of intraoperative cystoscopy. Obstet Gynecol. 2006;107:6:1366-1372.
4. Ribeiro S, Reich H, Rosenberg J, Guglielminetti E, Vidali A. The value of intra-operative cystoscopy at the time of laparoscopic hysterectomy. Hum Reprod. 1999;14:1727-1729.

5. Jelovsek JE, Chiung C, Chen G, Roberts S, Paraiso M, FalconeT. Incidence of lower urinary tract injury at the time of total laparoscopic hysterectomy. JSLS. 2007;11:4:422-427.

6. Johnson CM, Wilson DM, O'Fallon WM, Kurland LT. Renal stone epidemiology: a 25-year study in Rochester, MN. Kidney Int. 1979;16:624.

7. Stamatelou KK, Francis ME, Jones CA, Nyberg LM, Curhan GC. Time trends in reported prevalence of kidney stones in the United States: 1976-1994. Kidney Int 2003;63:1817. 